\title{
Variable-period permanent-magnet helical undulator
}

\author{
Jungho Mun, ${ }^{1,3}$ Young Uk Jeong, ${ }^{1, *}$ Nikolay A. Vinokurov, ${ }^{1,2}$ Kitae Lee, ${ }^{1}$ Kyu-Ha Jang, ${ }^{1}$ \\ Seong Hee Park, ${ }^{1}$ Min Yong Jeon, ${ }^{3}$ and Sang-In Shin ${ }^{4}$ \\ ${ }^{1}$ WCI Center for Quantum-Beam-based Radiation Research, Korea Atomic Energy Research Institute, \\ 1045 Daedeok-daero, Yuseong-gu, Daejeon, 305-353, Korea \\ ${ }^{2}$ Budker Institute of Nuclear Physics, Siberian Branch of Russian Academy of Sciences, \\ 11 Lavrentyev Prospect, Novosibirsk 630090, Russia \\ ${ }^{3}$ Department of Physics, Chungnam National University, 99 Daehak-ro, Yuseong-gu, Daejeon, \\ 305-764, Korea \\ ${ }^{4}$ Kyungin System, 399-8 Doryong-dong, Yuseong-gu, Daejeon, 305-340, Korea \\ (Received 13 July 2013; revised manuscript received 2 June 2014; published 1 August 2014)
}

\begin{abstract}
We realized a variable-period permanent-magnet helical undulator with high $(\sim 1 \mathrm{~T})$ field amplitude, which is almost constant over undulator periods of $23-26 \mathrm{~mm}$. Each undulator period has four modular sections of iron poles and permanent magnets embedded in nonmagnetic disks with holes along the undulator axis. Modular plates undergo a longitudinal repulsive force from the magnetic field pressure and the spring coils between modular plates. The undulator period can thus be controlled by mechanically changing the end plate longitudinal position. This compact design is suitable for tabletop terahertz free electron lasers.
\end{abstract}

DOI: 10.1103/PhysRevSTAB.17.080701

PACS numbers: 07.85.Qe, 41.60.Cr

\section{INTRODUCTION}

An undulator is a periodic magnetic (or electric) structure $[1,2]$. Undulators are used in free electron lasers (FELs) and spontaneous x-ray sources. Moving through the undulator ultrarelativistic electrons emit radiation. The wavelength of the undulator radiation in a forward direction is given by

$$
\lambda=\frac{\lambda_{w}}{2 \gamma^{2}}\left(1+K^{2}\right),
$$

where $\lambda_{w}$ is the undulator period and $\gamma$ is the Lorentz factor of a relativistic electron. The $K$ parameter takes into account the reduction of the longitudinal velocity of an electron due to the curvature of the wiggling trajectory. For a helical undulator $K \approx 0.934 B_{w}[\mathrm{~T}] \lambda_{w}[\mathrm{~cm}]$, where $B_{w}$ is the magnetic field on the undulator axis. In both FEL and spontaneous emission applications, it is necessary to tune the wavelength of undulator radiation.

Typically, variation of particle energy is impossible (on a storage ring with many undulators) or complicated (because of focusing and trajectory change). A frequently used way of undulator radiation wavelength tuning is variation of the field amplitude $B_{w}$. Serious drawbacks of this technique are too low field value at short wavelengths and too high one at long wavelengths. This circumstance is, in particular, an obstacle for using undulators with very short periods (and

\footnotetext{
‘vujung@kaeri.re.kr

Published by the American Physical Society under the terms of the Creative Commons Attribution 3.0 License. Further distribution of this work must maintain attribution to the author(s) and the published article's title, journal citation, and DOI.
}

therefore low $K$ ), because the tuning range of such undulators is very small. Wavelength tuning by field amplitude variation is especially difficult in x-ray FELs [3]. A decrease of field amplitude in these devices may cause a significant increase in the gain length. Moreover, the field amplitude must be the same in different sections of a long undulator, with a precision better than $0.1 \%$. It is especially difficult for variable-gap permanent magnet undulators.

Another solution for wavelength tuning is variation of the undulator period $\lambda_{w}$. The "simplest" way to do it is to replace one undulator with another [4,5]. In such a design, fine wavelength tuning is provided by gap variation. Different electromagnetic variable-period (V-P) undulators were discussed in Refs. [6-8]. The first two describe systems with fixed current distribution but moving ferromagnetic details. The last one proposes the period length switching by change of current distribution with fixed ferromagnetic yoke.

Recently, a permanent magnet planar V-P undulator was proposed [9]. It uses a split-pole structure of a hybrid permanent-magnet undulator. The moving units of two half-poles with permanent magnet between them undergo strong mutual repulsion. Therefore, distances between the units (half-periods) are almost equal, changing proportionally to the total length of the undulator. The V-P undulator gives almost constant field strength at different periods, which results in less variations of a gain and radiation power for a given wavelength tuning range as compared with those for undulators with variable field amplitude. As it is shown in Ref. [9], the V-P undulator has a far less stringent dimensional tolerance and less driving force as compared to those for the variable-gap undulator.

In this paper we describe another V-P permanent magnet undulator-the helical one. 
TABLE I. Main parameters of the V-P helical permanentmagnet undulator for a compact THz FEL.

\begin{tabular}{lc}
\hline \hline Gap (inner diameter), mm & 5 \\
Number of periods & 30 \\
Length of period, mm & $23-26$ \\
Peak magnetic field, T & 1 \\
\hline \hline
\end{tabular}

\section{DESIGN OF THE UNDULATOR}

It is difficult to introduce split-pole V-P geometry in a permanent-magnet undulator with a helical structure. We propose a new structure for a V-P permanent-magnet helical undulator by introducing magnetic and mechanical repulsive forces between adjacent modular plates of the undulator. The helical undulator may be considered as a superposition of two hybrid permanent-magnet planar undulators. If the magnetic field amplitudes and periods of the two planar undulators are equal and one

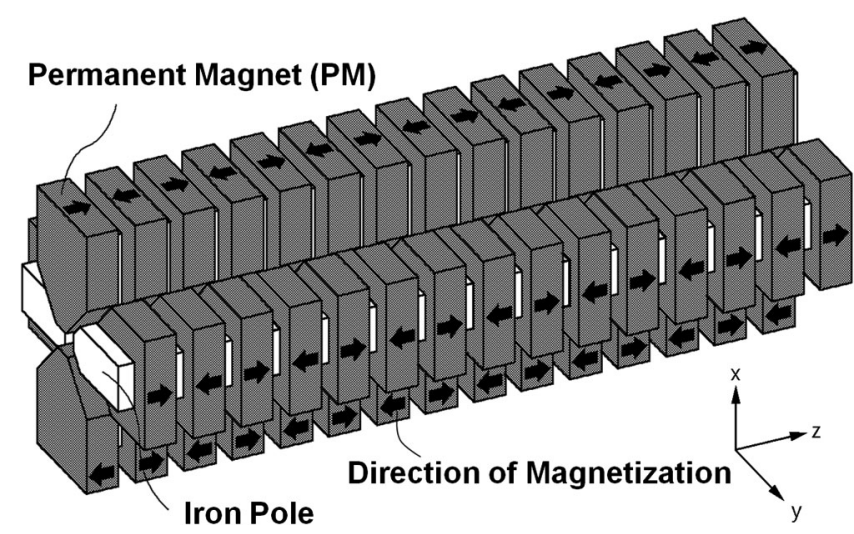

FIG. 1. Magnetic design of the V-P helical permanent-magnet undulator. The undulator is a combination of two planar hybrid permanent-magnet undulators. of them is shifted longitudinally by a quarter of a period with respect to another, a helical structure can be realized.

A three-dimensional (3D) simulation of the V-P helical undulator using the CST code [10] for different undulator period lengths was carried out. Table I shows the main parameters of our V-P helical undulator for a compact terahertz (THz) FEL. Figure 1 shows the structure of the helical undulator, consisting of permanent magnets and iron poles. This helical undulator is a combination of two planar undulators. For a given undulator gap $(5 \mathrm{~mm})$ and minimum period length $(23 \mathrm{~mm})$, the pole width was chosen to be $4 \mathrm{~mm}$ to maximize the magnetic field on the undulator axis. A short undulator with seven periods was then simulated for $\lambda_{\mathrm{w}}=23$ and $26 \mathrm{~mm}$. In this simulation, the sizes of the magnets and poles were fixed. Only the period length of the undulator was varied. Figure 2 shows the simulated vertical component of a magnetic field on the axis of the V-P helical undulator. According to the results of the simulation, the peak magnetic field on the undulator axis almost does not depend on the period length. Thus, the V-P helical undulator enables us to tune the FEL wavelength without significantly degrading the FEL gain. According to Eq. (1), the increase of the undulator period from 23 to $26 \mathrm{~mm}$ leads to the $40 \%$ increase of the radiation wavelength. This tunability range is enough for our compact THz FEL.

Each period of the undulator consists of four identical sections and each section has two iron poles and two permanent magnets embedded in a nonmagnetic disk, as shown in Fig. 3. The permanent magnets are fabricated from $\mathrm{NdFeB}$, which has a remanent flux density of about $1.2 \mathrm{~T}$. The modules are successively arranged with a relative rotation by $90^{\circ}$ about the undulator axis. As is clear from Fig. 4, the undulator is very simple and compact.

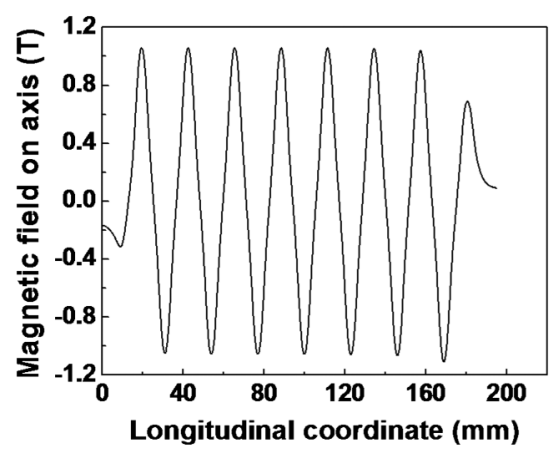

(a)

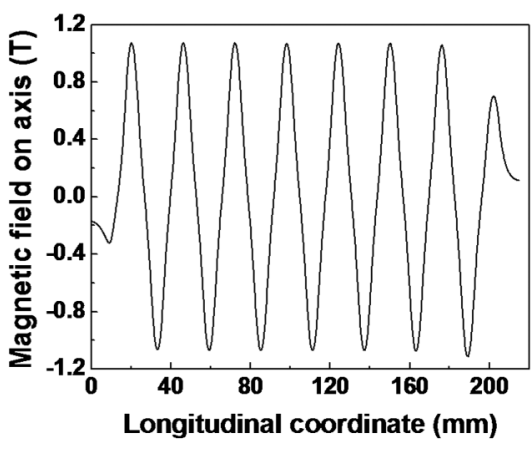

(b)

FIG. 2. Calculated vertical component of the on-axis magnetic field of the V-P helical undulator. The short undulator with seven periods was simulated for (a) $\lambda_{w}=23 \mathrm{~mm}$ and (b) $\lambda_{w}=26 \mathrm{~mm}$. The calculated peak magnetic field is about $1 \mathrm{~T}$. 


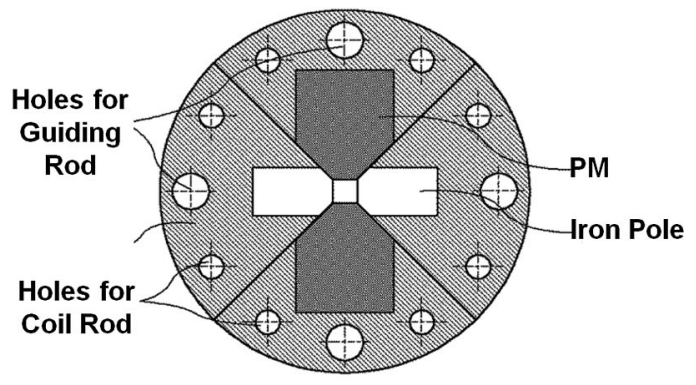

(a)

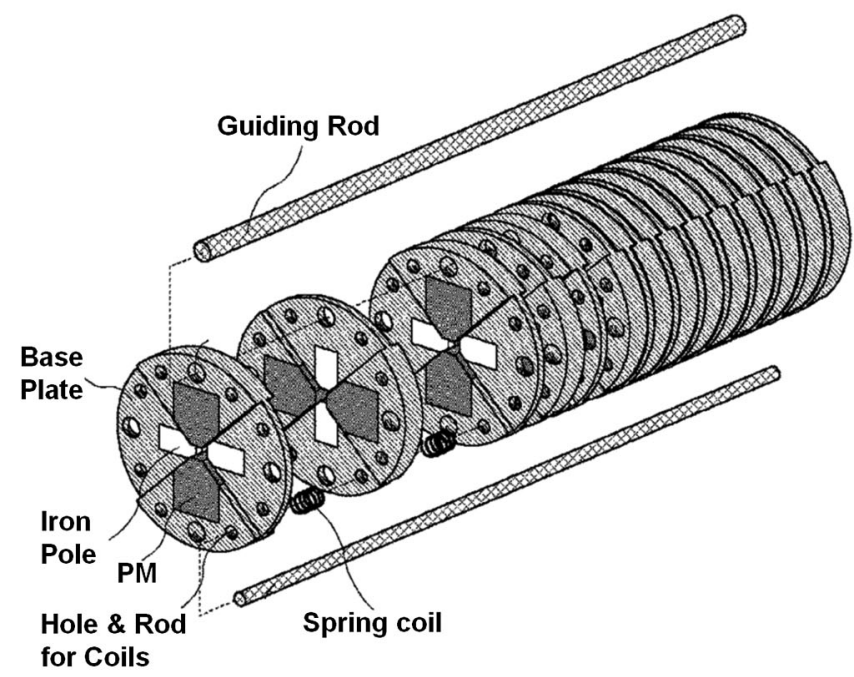

(b)

FIG. 3. (a) Schematics of a basic module of the V-P helical permanent-magnet undulator. The module contains two permanent magnets and two iron poles. (b) Assembly configuration of the undulator.

(a)

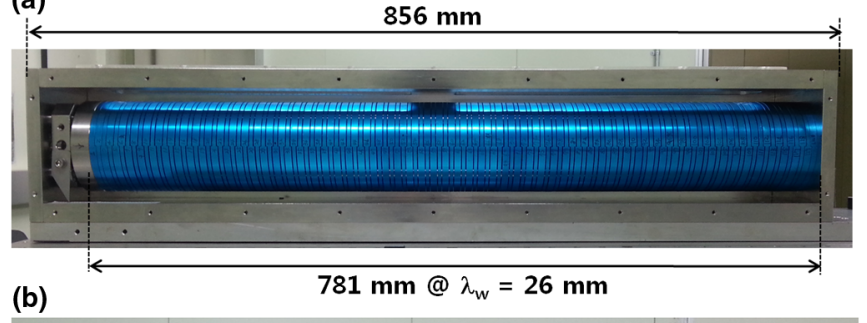

(b)

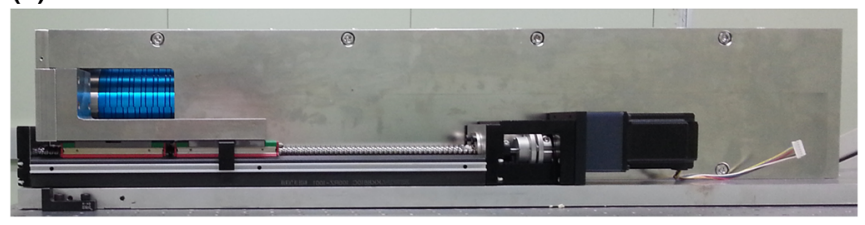

FIG. 4. Photographs of the V-P helical undulator. (a) Right side view of the undulator. Disk modules are azure. (b) Left side view of the undulator. Stepping motor and linear actuator are seen.

\section{LONGITUDINAL FORCES}

The distances between adjacent modules are almost equal due to the repulsive force between modules. This force is caused by the magnetic field pressure and springs.
The motion of the basic modules is constrained through nonmagnetic guide rods. By changing the longitudinal position of the last module with the motorized mechanical control, the total length of the undulator and, therefore, the period can be changed. The forces between magnet modules by the magnetic field $\mathbf{H}$ can be described by Maxwell's stress tensor [11] (in Gaussian units)

$$
\sigma_{i k}=\frac{1}{4 \pi}\left(H_{i} H_{k}-\frac{1}{2} H^{2} \delta_{i k}\right) .
$$

The repulsive force acting on a volume surrounded by a surface $S$ in vacuum is given by

$$
F_{i}=\oint_{S} \sigma_{i k} n_{k} d S,
$$

where $\mathbf{n}$ is the outer normal to the surface element $d S$. Equations (2) and (3) can be used for numerical calculations. To find the longitudinal force $F_{\mathrm{z}}$, it is convenient to choose the surface that divides the undulator into two halves, as shown in Fig. 5. The helical undulator contains four arrays of permanent magnets and iron poles. For part $S_{1}$ of the surface, which lies in the symmetry plane between the permanent magnets, the normal component $H_{\mathrm{z}}$ of the field is zero, and according to Eq. (2), $\sigma_{z z}=-H^{2} /(8 \pi)$. Magnetic pressure pushes the undulator halves away from each other. On the surface of the iron pole, the tangential field components are small. Therefore, on the area $S_{2}$, $\sigma_{z z}=H^{2} /(8 \pi)$, and an attractive contribution to the force is realized. On the side face of the pole $S_{3}, \sigma_{z x}=\sigma_{z y}=0$, and therefore $S_{3}$ does not contribute to the longitudinal force.

Thus, Eq. (3) gives

$$
F_{z}=-\int_{S_{1}} \frac{H^{2}}{8 \pi} d x d y+\int_{S_{2}} \frac{H^{2}}{8 \pi} d x d y .
$$

To estimate the first (repulsion) term, recall that modern magnetic materials have an almost constant magnetization

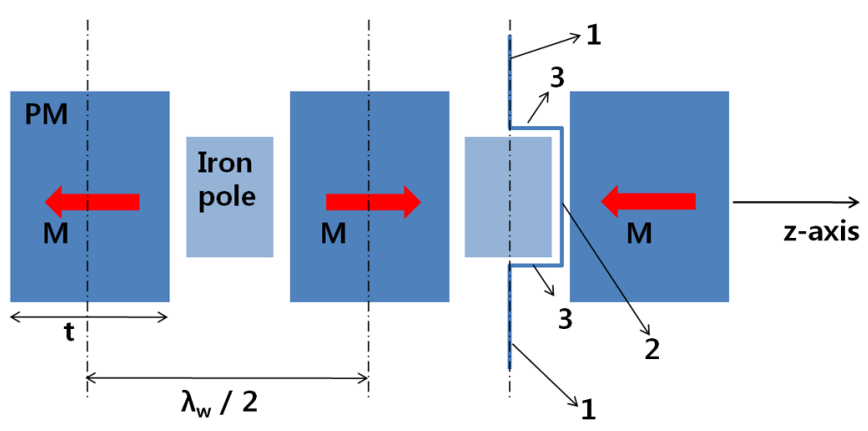

FIG. 5. Schematic diagram of magnet components for the estimation of repulsive forces between permanent magnets in the V-P helical undulator. 
(magnetic moment per unit volume) $\mathbf{M}$ that is almost independent of the field. In this case, the magnetization vector $\mathbf{M}$ is parallel or antiparallel to the $z$ axis. The resulting molecular current is then the surface electric current $\mathbf{i}=c \mathbf{M} \times \mathbf{n}$ on the side face of the magnet. Therefore, each magnetic block is equivalent to a current loop with electric current $I=i t=c M t$ ( $t$ is the magnetic block thickness) flowing along the permanent-magnet block perimeter $\Pi$. Through the definition $\mathbf{B}=\mathbf{H}+4 \pi \mathbf{M}$, the permanentmagnet material coercivity $H_{c}$, remanent field $B_{r}$, and magnetization $\mathrm{M}$ are connected by the relation $B_{r} \approx H_{c} \approx 4 \pi M$.

The force between two magnetic blocks can be calculated from the force between two current loops. If the distance between blocks (loops) is much less than the transverse size of a block, the force is just that between two linear parallel currents. This force can be expressed as

$$
F_{z}=\frac{I}{c} B \Pi=\frac{I}{c} \frac{2 I}{c a} \Pi=\frac{4 I^{2} \Pi}{c^{2} \lambda_{w}}=\frac{4 M^{2} t^{2} \Pi}{\lambda_{w}},
$$

where $a=\lambda_{w} / 2$ is the shift of one block with respect to another one. It may be shown by direct summation of forces for an infinite array of alternating magnetization of permanent-magnet blocks that the force between halves of an array is twice less than the value given by Eq. (5). By taking into account that in each module there are two such permanent magnets, one obtains the first term of Eq. (4)

$$
F_{z 1}=\frac{4 M^{2} t^{2} \Pi}{\lambda_{w}}=\frac{H_{c}^{2}}{8 \pi} \frac{2 t}{\lambda_{w}} \frac{t \Pi}{\pi},
$$

where $H_{c}$ is the coercivity of the permanent-magnet material. The first factor is the magnetic pressure corresponding to the coercivity value. In our case, for $H_{c}=12 \mathrm{kG}, \quad t=0.75 \mathrm{~cm}, \quad \Pi=9.345 \mathrm{~cm}, \quad$ and $\lambda_{w}=2.3 \mathrm{~cm}$, the repulsive force as estimated by Eq. (6) is about $100 \mathrm{~N}$. The second term in Eq. (4) and the (repulsive) contribution of the relatively high field $H_{g}$ in the undulator gap, $\sim \mathrm{g}^{2} H_{g}^{2} /(8 \pi)$, are significantly smaller than the main contribution given by Eq. (6). The inverse proportionality of the repulsive force Eq. (6) with undulator period provides mechanical stability. There are also eight springs between the magnet modules. The maximum repulsion force of each spring is $114 \mathrm{~N}$, and the spring rate is about $20 \mathrm{~N} / \mathrm{mm}$.

\section{FIELD DISTRIBUTION AND PERIODICITY MEASUREMENTS}

For analyzing the field distribution and periodicity of the V-P helical undulator, the field measurements were performed using a transverse Hall sensor (Lakeshore Inc.). The Hall probe was moved along the undulator axis using a linear motion stage. After the Hall probe was slowly inserted into the undulator gap, the field strength of the

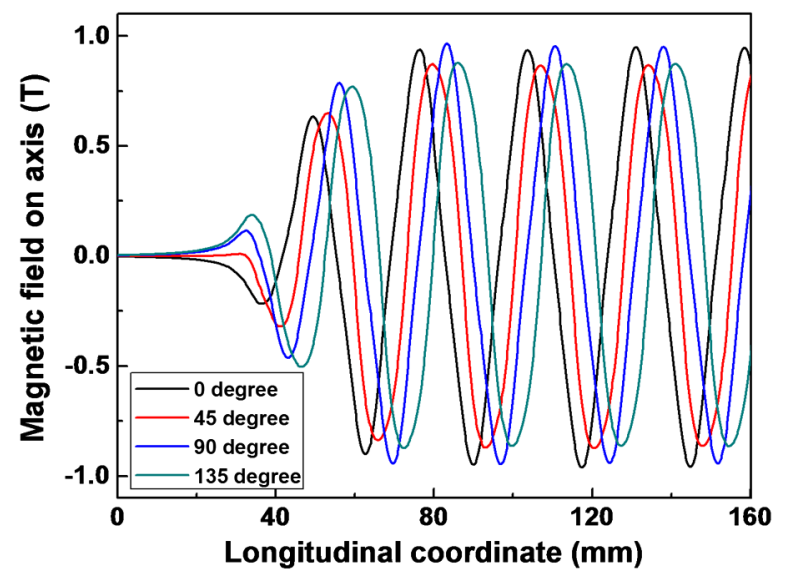

FIG. 6. Measured transverse component of field on the undulator axis at the period length of $26 \mathrm{~mm}$. Four curves correspond to different orientations of the Hall probe: redvertical, green $-45^{\circ}$ clockwise, blue $-90^{\circ}$ clockwise (i.e., horizontal), black- $135^{\circ}$ clockwise. The third harmonics of the undulator field decreases field maxima at skewed axes and increases them at normal (vertical and horizontal) ones (first and third harmonics rotate in opposite directions).

undulator was measured using a LabVIEW program (National Instrument Inc.). Figure 6 shows the measured values of the transverse field component on the undulator axis at a period length of $26 \mathrm{~mm}$. Four curves correspond to different orientations of the Hall probe: red-vertical, green$45^{\circ}$ clockwise, blue $-90^{\circ}$ clockwise (i.e., horizontal), black$135^{\circ}$ clockwise. The third harmonics of the undulator field decreases field maxima at skewed axes and increases them at normal (vertical and horizontal) ones (first and third harmonics rotate in opposite directions). To define precisely the field maxima, the raw data were filtered (very high and very low space frequencies were cut). The measured and simulated values are in good agreement. The measured average peak magnetic field is $0.96 \mathrm{~T}$. The difference of this value from the calculated one is less than the uncertainty of the permanent-magnet magnetization.

The new feature of this V-P undulator is the variable positions of poles and magnets. Therefore, it was interesting to measure the corresponding aperiodicity of the field. Figure 7 shows the measured results for the peak-to-peak lengths. We estimated these lengths through the analysis of distances between the maxima of the measured magnetic field component. The measurements were carried out for period lengths of $23.3,24,25$, and $25.6 \mathrm{~mm}$. The rms deviation of the peak-to-peak length in each case was less than $1 \%$. Figure 8 shows the dependence of the average fundamental harmonics of magnetic field on the period length of the V-P helical undulator. The inset graph of the figure shows the deviation of the peak magnetic field along the undulator axis for each period length. The rms deviation of the peak field module for each period length was measured to be less than $0.7 \%$. The first and the second 


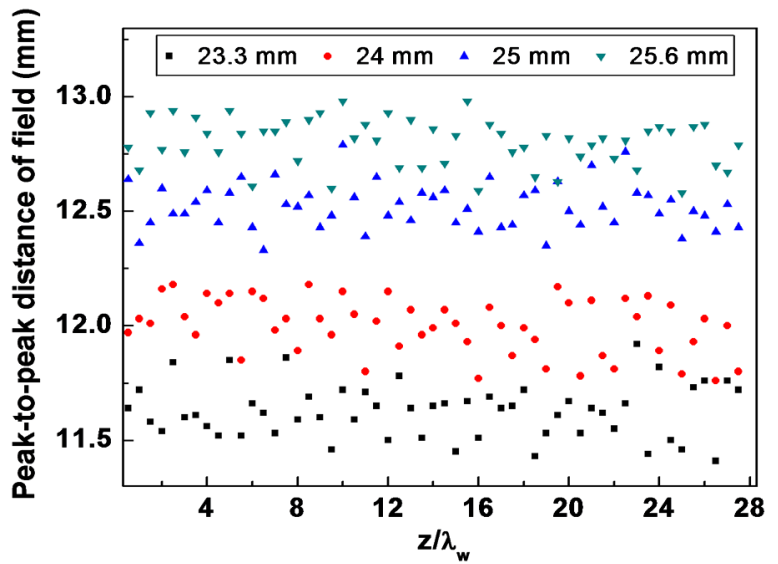

FIG. 7. Measured peak-to-peak distances for four undulator periods.

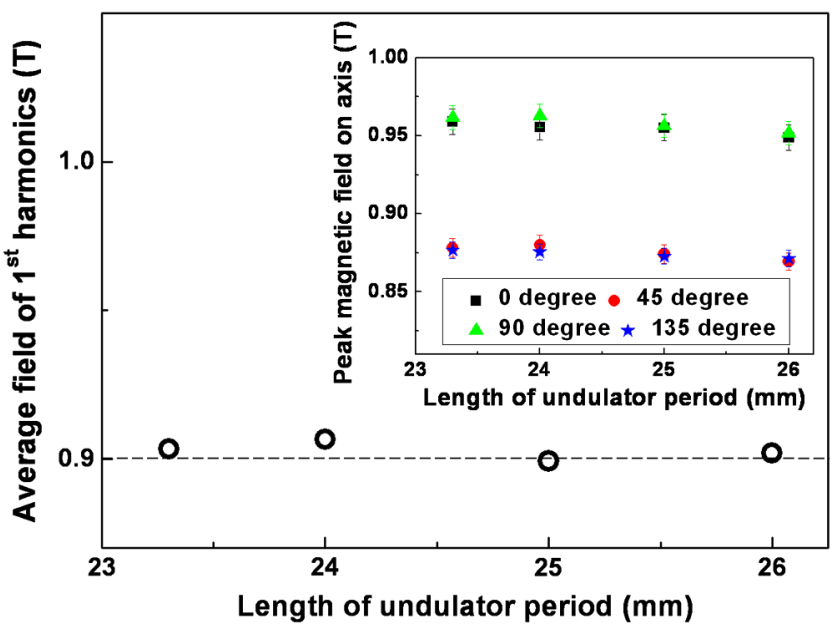

FIG. 8. Dependence of the average fundamental harmonics of magnetic field on the period length of the V-P helical undulator. The inset graph of the figure shows the peak magnetic field on the undulator axis.

field integrals and the corresponding radiation Fourier harmonics [2] were calculated. Calculated ratio of the maximum spectral intensity of radiation to the ideal one and rms phase errors are presented in Table II. Some reduction of the spectral intensity for the period $24 \mathrm{~mm}$ is caused by the period taper, which is seen in Fig. 7 . Moreover, it is seen in the plots of phase errors (Fig. 9).

TABLE II. Spectral intensity, normalized to the ideal one, and rms phase errors for different undulator periods.

\begin{tabular}{lcc}
\hline \hline $\begin{array}{l}\text { Period, } \\
\mathrm{mm}\end{array}$ & $\begin{array}{c}\text { Spectral } \\
\text { intensity } \\
\text { ratio }\end{array}$ & $\begin{array}{c}\text { rms phase errors, } \\
\text { degree }\end{array}$ \\
\hline 23.3 & $98 \%$ & 2.7 \\
24 & $95 \%$ & 8.0 \\
25 & $98 \%$ & 3.6 \\
25.6 & $97 \%$ & 3.7 \\
\hline \hline
\end{tabular}

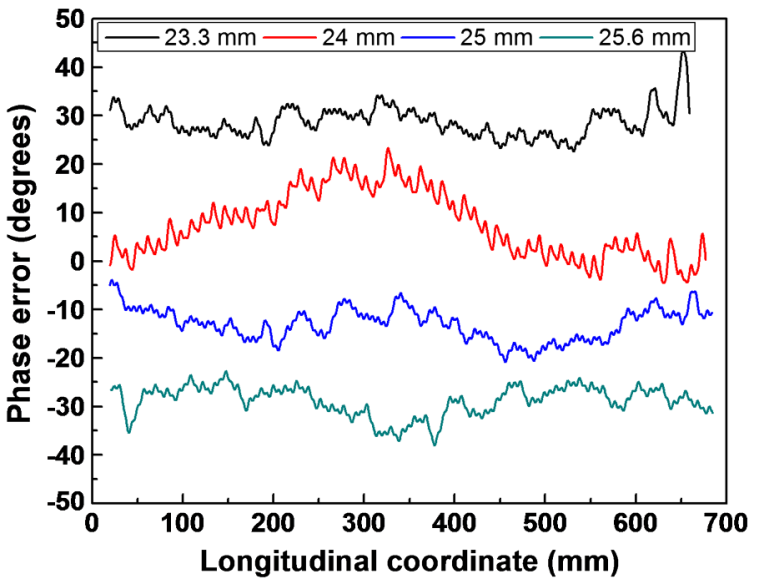

FIG. 9. Phase errors for periods 23.3, 24, 25, and $25.6 \mathrm{~mm}$ (top to bottom).

Probably, this tapering is caused by friction and was not reduced by back-and-forth motion of the actuator. The reproducibility of the phase error was checked by measuring the field 6 times at the $25.6 \mathrm{~mm}$ period after random shifts of undulator sections. The rms phase errors, calculated from these measurements are $4.14^{\circ}, 3.1^{\circ}, 2.99^{\circ}, 2.72^{\circ}$, $4.71^{\circ}$, and $4.56^{\circ}$. The mean value of the rms phase errors is 3.7 .

The results of these calculations show that the field errors are within the tolerances for a tabletop THz FEL. Further improvements of mechanical parts (especially, sliders) are necessary for longer V-P undulators.

\section{CONCLUSIONS}

To our knowledge, this is the first V-P permanentmagnet helical undulator described in this paper. This undulator has significant advantages. First, this design allows the tuning of the radiation wavelength by adjusting the undulator period without a decrease in the undulator field and, therefore, with less reduction in FEL gain. Second, the realization of a compact and mechanically simple system is possible with this design. Consequently, undulators of such type may be an interesting option for developing new FELs and cost-effective insertion devices for synchrotron radiation sources.

\section{ACKNOWLEDGMENTS}

This work was supported by the World Class Institute (WCI) Program of the National Research Foundation of Korea (NRF) funded by the Ministry of Science, ICT and Future Planning (NRF Grant No. WCI 2011-001).

[1] H. Onuki and P. Elleaume, Undulators, Wigglers and Their Applications (Taylor and Francis Inc., London, 2003). 
[2] E. Levichev and N. Vinokurov, Rev. Accel. Sci. Techol. 03, 203 (2010).

[3] T. Tanaka, Proceedings of the 31st International Free Electron Laser Conference (FEL 09), Liverpool, UK (STFC Daresbury Laboratory, Warrington, 2009), p. 524.

[4] R. Z. Bachrach, R. D. Bringans, B. B. Pate, and R. G. Carr, Proc. SPIE Int. Soc. Opt. Eng. 582, 251 (1985).

[5] G. Isoyama, S. Yamamoto, T. Shioya, J. Ohkuma, S. Sasaki, T. Mitsuhashi, T. Yamakawa, and H. Kitamura, Rev. Sci. Instrum. 60, 1863 (1989).

[6] A. van Steenbergen, J. Gallardo, T. Romano, and M. Woodle, Proceedings of the 1991 Particle Accelerator
Conference (APS Beams Physics) (IEEE, Piscataway NJ, 1991), p. 2724.

[7] G. K. Shenoy, J. W. Lewellen, D. Shu, and N. A. Vinokurov, J. Synchrotron Radiat. 10, 205 (2003).

[8] A. Bernhard, P. Peiffer, D. Wollmann, and T. Baumbach, Proceedings of the 11th European Particle Accelerator Conference, EPAC08, Genoa, Italy, (EPS-AG, Geneva, 2008), p. 2231.

[9] N. A. Vinokurov, O. A. Shevchenko, and V. G. Tcheskidov, Phys. Rev. ST Accel. Beams 14, 040701 (2011).

[10] CST EM Studio, 2005 CST-Computer Simulation Technology, Wellesley Hills, MA, www.cst.com.

[11] L. D. Landau and E. M. Lifshitz, The Classical Theory of Fields (Elsevier Science, Amsterdam, 1980). 